\title{
Competition between spin-orbit coupling and molecular orbital crystal in pyrochlore ruthenate $\mathrm{In}_{2} \mathrm{Ru}_{2} \mathrm{O}_{7}$
}

\author{
Aleksandra Krajewska ${ }^{1,2}$, Tomohiro Takayama ${ }^{2,3}$, Alexander Yaresko ${ }^{2}$, Jurgen Nuss ${ }^{2}$, Alexandra Gibbs ${ }^{1,2,4}$, \\ Sebastian Bette ${ }^{2}$, Hidenori Takagi ${ }^{2,3,5}$ \\ ${ }^{1}$ ISIS Neutron and Muon Source, Didcot, United Kingdom, ${ }^{2}$ Max Planck Institute for Solid State Research, Stuttgart, Germany, \\ ${ }^{3}$ Institute for Functional Matter and Quantum Technologies, University of Stuttgart, Stuttgart, Germany, ${ }^{4}$ School of Chemistry, \\ University of St Andrews, St Andrews, United Kingdom, ${ }^{5}$ Department of Physics, University of Tokyo, Tokyo, Japan \\ aleksandra.krajewska@stfc.ac.uk
}

Transition metal oxides are a platform for a plethora of exotic electronic phases where multiple degrees of freedom of correlated delectrons, together with an underlying lattice topology, are at play. The ground states of these systems are governed by a subtle balance of relevant electronic parameters such as Coulomb repulsion, bandwidth, and crystal fields. $4 d$ ruthenium compounds have been playing a significant role in providing novel electronic states such as unconventional superconductivity, metal-insulator transition, and quantum magnetism.

Besides metallic or Mott insulating ground states, some ruthenium compounds exhibit a nonmagnetic ground state, which is accompanied by the formation of molecular orbitals generated by direct hopping between spatially extended $4 d$ orbitals. A prominent example is the honeycomb ruthenate $\mathrm{Li}_{2} \mathrm{RuO}_{3}$, which undergoes dimerization of $\mathrm{Ru}$ atoms below $\sim 550 \mathrm{~K}$ and forms a "molecular orbital crystal", where the $4 d$ electrons are accommodated into the bonding and antibonding molecular orbitals localized on the dimers.

In heavy-transition-metal compounds such as ruthenates, another key ingredient for their exotic electronic states is spin-orbit coupling (SOC) which produces spin-orbit entangled $J_{\text {eff }}$ pseudospins. Probably the most striking impact on magnetism is realised in $\mathrm{Ru}^{4+}$ ruthenates with a $d^{4}$ configuration. While SOC produces a nominally non-magnetic $J_{\text {eff }}=0$ singlet, "excitonic" magnetism can arise via the interaction between excited states, and spin-orbit excitons may condense into an exotic long-range magnetic order. Up to date, excitonic magnetism has been only established in a layered perovskite $\mathrm{Ca}_{2} \mathrm{RuO}_{4}$ and remains unexplored in other ruthenates.

The competition between electronic phases including molecular orbital crystal and spin-orbit magnetism is expected to be more pronounced in ruthenates with a frustrated lattice, such as pyrochlore ruthenates $A_{2} \mathrm{Ru}_{2} \mathrm{O}_{7}(A$ : trivalent cation). The pyrochlore ruthenates have been regarded as $S=1$ Mott insulators due to the presence of a trigonal distortion which may lift the degeneracy of the $t_{2 \mathrm{~g}}$ orbitals and thus competes with SOC. While most of them order magnetically at low temperatures, $\mathrm{Tl}_{2} \mathrm{Ru}_{2} \mathrm{O}_{7}$ exhibits a metal to non-magnetic insulator transition at $\sim 120 \mathrm{~K}$. The origin of the nonmagnetic ground state has been attributed to the formation of a Haldane gap in the one-dimensional zigzag chains of Ru atoms on top of the pyrochlore lattice. The distinct behaviour of $\mathrm{Tl}_{2} \mathrm{Ru}_{2} \mathrm{O}_{7}$ may be related to the covalency of Tl-O bonds, which has been discussed as playing a key role in the metal-insulator transition. The covalent character of $A$-O bonds thus may be an important parameter for the ground state of pyrochlore oxides. On the other hand, the role of spin-orbit coupling has not been fully investigated in pyrochlore ruthenates.

In an attempt to explore the novel phase competition in pyrochlore ruthenates, we discovered a new compound $\operatorname{In}_{2} \mathrm{Ru}_{2} \mathrm{O}_{7}$ using high pressure synthesis. At high temperatures above $\sim 450 \mathrm{~K}, \mathrm{In}_{2} \mathrm{Ru}_{2} \mathrm{O}_{7}$ crystallizes in a cubic pyrochlore structure, but adopts a weakly distorted tetragonal structure at room temperature as elucidated with single crystal $\mathrm{x}$-ray and powder neutron diffraction. From the spectroscopic measurements, $\mathrm{In}_{2} \mathrm{Ru}_{2} \mathrm{O}_{7}$ was found to host a spin-orbit-entangled $J_{\text {eff }}=0$-like state at room temperature, despite presenting the largest trigonal distortion among the family of pyrochlore ruthenates. The spin-orbit entangled singlet state is expected to display excitonic magnetism. Strikingly, through successive structural transitions likely associated with the covalent In-O bonds, the singlet state collapses and $\operatorname{In}_{2} \mathrm{Ru}_{2} \mathrm{O}_{7}$ forms a nonmagnetic state below $\sim 220 \mathrm{~K}$ as evidenced by muon spin rotation. The nonmagnetic ground state was found to originate from a molecular orbital formation in the semi-isolated $\mathrm{Ru}_{2} \mathrm{O}$ trimer molecules decorating the pyrochlore lattice. Such molecular orbital formation, which involves not only the $\mathrm{Ru}^{4+}$ ions but the $\mathrm{O}^{2-}$ anions as well, has not been reported in other pyrochlore oxides.

In this talk we discuss the subtle competition between spin-orbit coupling and molecular orbital crystal formation in pyrochlore ruthenate $\mathrm{In}_{2} \mathrm{Ru}_{2} \mathrm{O}_{7}$. We present the structural details of the $\mathrm{Ru}_{2} \mathrm{O}$ trimer formation and its impact on the magnetism and electronic structure of $\mathrm{In}_{2} \mathrm{Ru}_{2} \mathrm{O}_{7}$. We argue that the unique molecular orbital formation involving an oxygen atom, distinct from dimers with direct overlap of $d-$ orbitals commonly found in other transition metal oxides, is assisted by the distortion of the In-O network. Our result demonstrates that bond covalency of constituent ions can be an additional key parameter in understanding phase competitions in complex transitionmetal oxides.

Keywords: correlated materials, ruthenate, structural phase transition, spin-orbit coupling, molecular orbital crystal 